\title{
Dissection of Molecular Mechanisms Regulating Protein Body Formation in Maize Endosperm - DE-FG03-95-ER20183
}

\author{
Final Technical Report and Patent Summary
}

\author{
Dr. Brian A. Larkins, Department of Plant Sciences, University of Arizona, Tucson, AZ \\ 85721
}

Endosperm texture is an important quality trait in maize, as it influences the shipping characteristics of the grain, its susceptibility to insects, the yield of grits from dry milling, energy costs during wet milling, and the baking and digestibility properties of the flour. There appears to be a causal relationship between kernel hardness and the formation of zein-containing protein bodies, as mutations affecting protein body number and structure are associated with a soft, starchy kernel. In this project we used a variety of approaches to better understand this relationship and investigate the molecular and biochemical changes associated with starchy endosperm mutants. We characterized the distribution of zein mRNAs on endosperm rough endoplasmic reticulum (RER) membranes and the interactions between zein proteins, as each of these could influence the structure of protein bodies. Based on in situ hybridization, mRNAs encoding the $22-\mathrm{kD}$ alpha- and 27-kD gamma-zeins are randomly distributed on RER; hence, mRNA targeting does not appear to influence the formation of protein bodies. Investigation of the interactions between zein proteins (alpha, beta, gamma, delta) with the yeast two-hybrid system showed that interactions between the 19- and 22-alpha-zeins are relatively weak, although each of them interacted strongly with the $10-\mathrm{kD}$ delta-zein. Strong interactions were detected between the alpha- and delta-zeins and the 16-kD gamma- and 15-kD betazeins; however, the 50-kD and 27-kD gamma-zeins did not interact detectably with the alpha- and delta-zein proteins. The $\mathrm{NH}_{2}$ - and $\mathrm{COOH}$-terminal domains of the 22-kD alpha-zein were found to interact most strongly with the $15-\mathrm{kD}$ beta- and $16-\mathrm{kD}$ gammazeins, suggesting the $16-\mathrm{kD}$ and $15-\mathrm{kD}$ proteins bind and assemble alpha-zeins in protein bodies. Additional evidence supporting this hypothesis was obtained by showing that the starchy endosperm mutant, Mucuronate, appears to result from a defective 16-kD gamma-zein protein. By deletion mutagenesis, we identified domains within an alphazein that cause it to interact with other zein proteins, particularly gamma-zeins. This allowed us to develop a minimal alpha-zein gene construct that can be used as a vector to target heterologous proteins, such as green fluorescent protein, into protein bodies. We characterized the nature of storage proteins synthesized in the endosperm using a genomics analysis of endosperm ESTs. This study identified several new storage proteins and demonstrated the existence of novel protein storage vacuoles. We used mRNA transcript profiling of eight different starchy endosperm (opaque) mutants (ol, o2, o5, o9, o1 1, Mucronate, Defective endosperm B30, and floury2) to identify patterns of gene expression that are consistently altered in all of them, or that are unique to each one of them. These mutants fall into two subgroups: one systematically manifests an "unfolded protein" response $(f l 2, M c, D e B 30)$ and the other $(o 1, o 2, o 5, o 9, o 11)$ does not. Genes encoding cytoskeletal proteins are generally up-regulated in all the mutants, and this may be associated with higher lysine contents in several of them. 